\title{
PERSPECTIVE SIMPLEXES AND TUCKER QUADRICS
}

\author{
AUGUSTINE O. KONNULLY
}

(Received 18 March 1970; revised 12 November 1970)

Communicated by G. Szekeres

\section{Introduction}

The sides of a triangle are met by the sides of a triangle in perspective with it in nine points of which three lie on the axis of perspectivity of the triangles and the other six, as we have it from the converse of Pascal's theorem, lie on a conic. When the centre of perspectivity is the Lemoine point of the triangle and the axis of perspectivity is the line at infinity this conic is a circle known as a Tucker circle [1]. In this paper, analogously we prove that given a simplex in $n$-space, the traces on its edges of the prime faces of any simplex in perspective with it, all except those falling on the prime of perspectivity, lie on a quadric which we call a Tucker quadric. And further we consider the special case when this quadric becomes a hypersphere.

\section{Simplexes perspective from the same point and prime}

Let $S$ be a simplex in $n$-space. Let this be the simplex of reference with vertex-vectors $\boldsymbol{e}_{0}, \boldsymbol{e}_{1}, \cdots, \boldsymbol{e}_{n}$. Denote the face opposite the vertex $\boldsymbol{e}_{i}$ by $\boldsymbol{\varepsilon}_{i}$. If $\boldsymbol{y}=\sum_{i=0}^{n} y_{i} e_{i}=\left(y_{0}, y_{1}, \cdots, y_{n}\right)$ is any point disjoint from $S$, we shall write $y_{i}{ }^{*}=y_{i}{ }^{-1}$ and $\boldsymbol{y}^{*}=\left(y_{0}{ }^{*}, y_{1}{ }^{*}, \cdots, y_{n}{ }^{*}\right)$. Let $A$ be a simplex with vertices $\boldsymbol{a}_{i}$, $(i=0,1, \cdots, n)$ which is perspective with $S$ from a point $\boldsymbol{u}$. Then $\boldsymbol{a}_{i}=\boldsymbol{u}+k_{i} \boldsymbol{e}_{i}$ for some $k_{0}, k_{1}, k_{2}, \cdots, k_{n}$, and the face $\alpha_{i}$ of $A$ opposite the vertex $\boldsymbol{a}_{i}$ is the prime $\boldsymbol{k}^{* T} \boldsymbol{x}-g x_{i} / u_{i}=0$, where $g=\boldsymbol{k}^{* T} \boldsymbol{u}+1$. Hence $\varepsilon_{i}$ and $\alpha_{i}$ meet on the prime $\kappa$ with equation $k^{* T} x=0$, which is thus the prime of perspectivity.

Any simplex perspective with $S$ from the same point $\boldsymbol{u}$, having vertices say $\boldsymbol{u}+\boldsymbol{k}^{\prime} \boldsymbol{e}_{i}$, will have the prime of perspectivity $\kappa$ if and only if $\boldsymbol{k}^{\prime}=\boldsymbol{t} \boldsymbol{k}$ for some scalar $t$. Denote the simplex with vertices $u+t k_{i} e_{i}$ by $A(t)$. The simplexes $A(t)$ form a system $(A)$ any two of which have centre of perspective $\boldsymbol{u}$ and axis of perspective $\kappa$.

\section{Antiparallel simplexes}

The prime face $\alpha_{i}(t)$ of $A(t)$ opposite the vertex $a_{i}(t)=u+t k_{i} e_{i}$ meets the edges of $S$ other than those issuing from the vertex $\boldsymbol{e}_{i}$ in points which lie on $\kappa$. 
Consider the points in which $\alpha_{i}(t)$ meets the edges issuing from $e_{i}$. Let

$$
p_{j}^{i}(t)=a_{i}(t) \cap\left\langle e_{i}, e_{j}\right\rangle, j \neq i .
$$

There are altogether $n(n+1)$ points $\boldsymbol{p}_{j}^{i}(t)$, two on each edge $\left\langle\boldsymbol{e}_{i}, \boldsymbol{e}_{j}\right\rangle$.

Let $\beta_{j}(t)$ be the prime containing the points $p_{j}^{h},(h=0,1, \ldots, j-1, j+1, \ldots, n)$, and let $B(t)$ be the simplex with faces $\beta_{0}(t), \beta_{1}(t), \cdots, \beta_{n}(t)$. We call $B(t)$ the antiparallel simplex of $A(t)$ with respect to $S, \boldsymbol{u}$ and $\kappa$.

It is easily seen that

$$
\boldsymbol{p}_{j}^{i}(t)=\frac{u_{i} k_{i}}{k_{i} g(t)-u_{1}} \boldsymbol{e}_{i}+k_{j} \boldsymbol{e}_{i}, \text { where } g(t)=\boldsymbol{k}^{* r} \boldsymbol{u}+t,
$$

and that $\beta_{i}(t)$ is the prime

$$
\sum_{j=0}^{n} \frac{k_{j} g(t)-u_{j}}{u_{j} k_{j}} x_{j}-g(t) x_{i} / u_{i} \equiv g(t)\left\{\boldsymbol{u}^{* T} \boldsymbol{x}-x_{i} / u_{i}\right\}-\boldsymbol{k}^{* T} \boldsymbol{x}=0 .
$$

The vertices of $B(t)$ are $u+c_{j} e_{j}$, where

$$
c_{j}=k_{j} u_{j}(t+\overline{n-1} g(t)) /\left(u_{j}-k_{j} g(t)\right) .
$$

So $B(t)$ is in perspective with all the simplexes $A\left(t^{\prime}\right)$ from $u$. Since $\alpha_{i}\left(t^{\prime}\right)$ is the prime

$$
\boldsymbol{k}^{* T} \boldsymbol{x}-g\left(t^{\prime}\right) x_{i} / u_{i}=0,
$$

the prime of perspectivity for $B(t)$ and $A\left(t^{\prime}\right)$ is

$$
g(t) g\left(t^{\prime}\right) \boldsymbol{u}^{* T} x-\left\{g(t)+g\left(t^{\prime}\right)\right\} \boldsymbol{k}^{* T^{\prime}} x=0 .
$$

So also any two simplexes $B(t)$ are perspective from $u$ and have prime of perspectivity $\kappa$. Thus

THEOREM 1. The simplexes $B(t)$ form a system $(B)$, any two of the simplexes being in perspective from $\boldsymbol{u}$ and $\kappa$. Any simplex $B(t)$ is in perspective with any simplex $A\left(t^{\prime}\right)$ from the point $\boldsymbol{u}$ and $\alpha$ (variable) prime through the intersection of $\kappa$ and $\boldsymbol{u}^{* T} \boldsymbol{x}=0$.

\section{Tucker quadrics}

THEOREM 2. Let $S$ and $T$ be two simplexes in $n$-space which are in perspective. The points in which the prime faces of one simplex meet the edges of the other, except those lying in the prime of perspectivity $\kappa$, lie on a quadric; and this quadric passes through the intersection of $\kappa$ and the polar quadric with respect to $S$ of the centre of perspective $u$.

Proof. In the previous notation, take $T=A(t)$. The points in question, namely the $n(n+1)$ points $p_{j}^{l}(t)$, are easily seen to lie on the quadric

$$
g(t)^{2} F_{u}-k^{* T} x\left\{g(t) u^{* T} x-k^{* T} x\right\}=0,
$$


where $F_{u} \equiv \frac{1}{2} \Sigma_{i \pm j} x_{i} x_{j} / u_{i} u_{j}=0$ is the polar quadric $[2,3]$ of $\boldsymbol{u}$ with respect to $S$.

Definition. The quadrics (4) are called the Tucker quadrics of $S$ relative to $u$ and $\kappa$.

We note that, if $v$ is the pole of $\kappa$ with respect to the quadric $F_{u}=0$, then the poles of $\kappa$ with respect to the various Tucker quadrics all lie on the fixed line $\langle\boldsymbol{u}, \boldsymbol{v}\rangle$, as do the poles of $\boldsymbol{u}^{* T} \boldsymbol{x}=0$ (the polar prime of $\boldsymbol{u}$ with respect to $S$ ).

Some special cases of Theorem 2 are worth mentioning. Suppose $S$ is isodynamic, that is, the circumhypersphere of $S$ is the polar quadric of some point, the Lemoine point of $S[3,4]$. Let $\boldsymbol{u}$ be the Lemoine point and $\kappa$ the absolute prime. Then $F_{u}=0$ is the circumhypersphere of $S$ and its intersection with $\kappa$ is the absolute $(n-2)$-dimensional quadric. The simplexes $A(t)$ are homothetic to $S$, and every Tucker quadric of $S$ relative to $u$ and $\kappa$, passing as it does through the intersection of $F_{u}=0$ and $\kappa$, is a hypersphere.

In this case, any face $\beta_{i}(t)$ of $B(t)$ is parallel to the tangent hyperplane, of the circumhypersphere of $S$, at the vertex $e_{i}$ of $S$. We say $\beta_{i}(t)$ is antiparallel to the face $\varepsilon_{i}$ of $S$.

When $t \rightarrow 0$, the vertices of $A(t)$ will all coincide with $u$ and the faces of $A(t)$ become the primes parallel to the faces of $S$ through $u$. The corresponding Tucker hypersphere may be called the first Lemoine hypersphere of $S$, in analogy with the circle obtained in 2-space.

Again, when $t$ takes the value which makes $(n-1) g(t)+t=0$, the vertices of the antiparallel simplex $B(t)$ all coincide with $u$ and the faces become the antiparallels, through $u$, to the faces of $S$. The corresponding Tucker hypersphere might be called the second Lemoine hypersphere of $S$. Its centre is at the Lemoine point $\boldsymbol{u}$ of $S$, since $\boldsymbol{u}$ and $\kappa$ are polars with respect to the Tucker quadric when $(n-1) g(t)+t=0$.

My thanks are due to the referee for helpful suggestions.

\section{References}

[1] N. A. Court, College Geometry (Barnes and Noble Inc., New York, 1952).

[2] J. G. Semple and L. Roth, Algebraic Geometry (Oxford, 1949).

[3] S. R. Mandan; 'Polarity for a simplex', Czech. Math. Jour. 16 (91) (1966), 307-313.

[4] S. R. Mandan; 'Isodynamic and Isogonic Simplexes', (To Enrico Bompiani on his scientific jubilee) Ann. Mat. pura ed appl. (IV) 53 (1961), 54-56.

St. Albert's College, Ernakulam

Cochin (India) 